\title{
Protective Role of Endogenous Kallistatin in Vascular Injury and Senescence by Inhibiting Oxidative Stress and Inflammation
}

\author{
Julie Chao $(D$, Youming Guo, and Lee Chao \\ Department of Biochemistry and Molecular Biology, Medical University of South Carolina, Charleston, SC, USA \\ Correspondence should be addressed to Julie Chao; chaoj@musc.edu
}

Received 28 June 2018; Accepted 4 October 2018; Published 2 December 2018

Academic Editor: Roberto Carnevale

Copyright (c) 2018 Julie Chao et al. This is an open access article distributed under the Creative Commons Attribution License, which permits unrestricted use, distribution, and reproduction in any medium, provided the original work is properly cited.

\begin{abstract}
Kallistatin was identified in human plasma as a tissue kallikrein-binding protein and a serine proteinase inhibitor. Kallistatin exerts pleiotropic effects on angiogenesis, oxidative stress, inflammation, apoptosis, fibrosis, and tumor growth. Kallistatin levels are markedly reduced in patients with coronary artery disease, sepsis, diabetic retinopathy, inflammatory bowel disease, pneumonia, and cancer. Moreover, plasma kallistatin levels are positively associated with leukocyte telomere length in young African Americans, indicating the involvement of kallistatin in aging. In addition, kallistatin treatment promotes vascular repair by increasing the migration and function of endothelial progenitor cells (EPCs). Kallistatin via its heparin-binding site antagonizes TNF- $\alpha$-induced senescence and superoxide formation, while kallistatin's active site is essential for inhibiting miR-34a synthesis, thus elevating sirtuin 1 (SIRT1)/eNOS synthesis in EPCs. Kallistatin inhibits oxidative stress-induced cellular senescence by upregulating Let-7g synthesis, leading to modulate Let-7g-mediated miR-34a-SIRT1-eNOS signaling pathway in human endothelial cells. Exogenous kallistatin administration attenuates vascular injury and senescence in association with increased SIRT1 and eNOS levels and reduced miR-34a synthesis and NADPH oxidase activity, as well as TNF- $\alpha$ and ICAM- 1 expression in the aortas of streptozotocin- (STZ-) induced diabetic mice. Conversely, endothelial-specific depletion of kallistatin aggravates vascular senescence, oxidative stress, and inflammation, with further reduction of Let-7g, SIRT1, and eNOS and elevation of miR-34a in mouse lung endothelial cells. Furthermore, systemic depletion of kallistatin exacerbates aortic injury, senescence, $\mathrm{NADPH}$ oxidase activity, and inflammatory gene expression in STZ-induced diabetic mice. These findings indicate that endogenous kallistatin displays a novel role in protection against vascular injury and senescence by inhibiting oxidative stress and inflammation.
\end{abstract}

\section{Introduction}

Aging is a major risk factor for the development of many diseases, including cardiovascular disease, stroke, and cancer [13]. Endothelial aging is associated with increased oxidative stress and inflammation and decreased endothelial nitric oxide synthase (eNOS) activity and nitric oxide $(\mathrm{NO})$ production $[4$, 5]. Excessive oxidative stress and chronic inflammation are common causes of endothelial dysfunction in vascular disease and aging [4]. Oxidative stress impairs the mobility and function of endothelial progenitor cells (EPCs) and enhances cellular senescence [6]. EPCs play an integral role in vascular repair by the replenishment of damaged or senescent endothelial cells [7]. Circulating EPC number and function are markedly reduced in the aging population [8-11]. Kallistatin in human plasma has been identified as a tissue kallikrein inhibitor and a unique serine proteinase inhibitor [12-16]. Kallistatin exerts multifactorial activities, including vasodilation and inhibition of oxidative stress, inflammation, fibrosis, and apoptosis, primarily by increasing eNOS levels and NO formation [17-22]. Moreover, kallistatin administration increases circulating EPC number and reduces aortic oxidative stress, whereas kallistatin depletion augments endothelial cell loss, diminishes circulating EPC levels, and exacerbates renal and cardiovascular oxidative stress, inflammation, and organ remodeling in hypertensive rats $[23,24]$. Kallistatin protein treatment enhances the migration and function of cultured human EPCs [23]. Furthermore, plasma kallistatin levels are positively associated with leukocyte telomere length in young African Americans [25]. Telomere length is critically related to vascular cell senescence, 


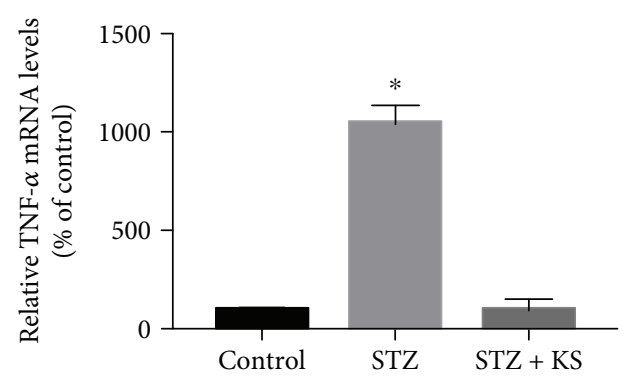

(a)

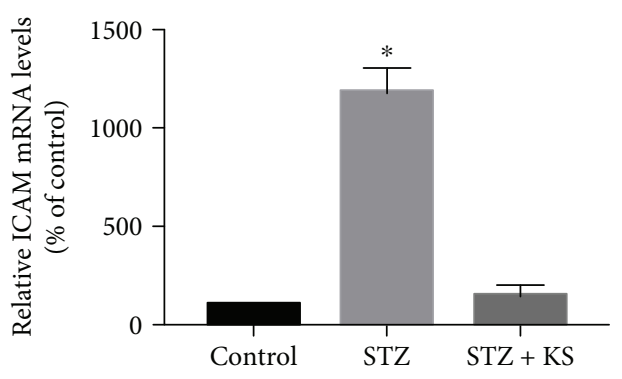

(b)

FIGURE 1: Kallistatin treatment on TNF- $\alpha$ and ICAM-1 synthesis in the aorta of STZ-induced diabetic mice. Values are expressed as mean \pm SEM. $n=3 .{ }^{*} P<0.05$ vs. the control group.

and NO prevents telomere shortening by stimulating telomerase activity [26, 27]. Therefore, kallistatin via NO formation may protect against vascular injury, senescence, and aging through its antioxidant and anti-inflammatory actions.

\section{Reduced Kallistatin Levels in Vascular Disease and Metabolic Disorders}

Kallistatin is mainly synthesized in the liver and distributed in blood vessels and organs relevant to cardiovascular function $[14,28-30]$. Human kallistatin gene transcripts and protein can be detected in the plasma, liver, heart, lung, kidney, prostate gland, and aorta $[14,28,29]$. Kallistatin is localized in endothelial and smooth muscle cells of large, medium, and small blood vessels [30]. Kallistatin levels are reduced in a variety of human diseases, such as coronary artery disease, sepsis, severe pneumonia, and active pulmonary tuberculosis, as well as colon, prostate, and liver cancer [29, 31-35]. Moreover, reduced plasma kallistatin levels are associated with elevated obesity and cardiometabolic risk in apparently healthy African Americans [36]. Likewise, kallistatin levels are lower in vitreous fluids from patients with diabetic retinopathy and in the retinas of streptozotocin- (STZ-) induced diabetic rats [37, 38]. However, elevated serum kallistatin has been observed in patients with diabetic vascular complications [39]. Moreover, plasma kallistatin levels are markedly reduced in animal models of hypertension, STZ-induced diabetes mellitus, lipopolysaccharide- (LPS-) induced endotoxemia, renal injury, and hepatocellular carcinoma [15, 18, 29, 4042]. Circulating kallistatin levels are negatively associated with elevated thiobarbituric acid reactive substance (TBARS, an indicator of oxidative stress) in diabetic rats [43]. In addition, kallistatin levels are reduced in animal models and humans with inflammatory disorders [29, 33, 44]. These combined findings indicate that kallistatin levels are reduced under excess oxidative stress and inflammation, implicating the involvement of kallistatin in vascular damage and metabolic disorders.

\section{Kallistatin as an Effective Antioxidant and Anti-Inflammatory Agent}

Oxidative stress is the main cause of endothelial injury and vascular disease states, and inflammation is known to increase with aging [45, 46]. Kallistatin administration attenuates hypertension and organ damage in conjunction with increased eNOS and NO levels and reduced oxidative stress and inflammation in animal models [18, 19, 22, 23]. Kallistatin administration markedly reduces inflammatory responses in animal models of arthritis, hypertension, myocardial ischemia, and septic shock $[17,19,21,22,47,48]$. Moreover, kallistatin overexpression protects against diabetic retinopathy in $d b / d b$ mice by multiple mechanisms, including antioxidant, anti-inflammatory, antifibrotic, and blood pressure-lowering effects [49]. NO has antioxidant properties by inhibiting NADPH oxidase activity [50]. Kallistatin, via $\mathrm{NO}$ formation, reduces $\mathrm{H}_{2} \mathrm{O}_{2}$ - or angiotensin II-induced NADPH oxidase activity and reactive oxygen species (ROS) formation in cultured endothelial cells, renal tubular cells, and cardiomyocytes [18, 22, 47]. Kallistatin inhibits vascular inflammation and apoptosis by stimulating eNOS synthesis and activation and NO formation [22, 51], as well as by preventing TNF- $\alpha$ - and high-mobility group box protein 1- (HMGB1-) mediated inflammatory gene expression [17, 21]. Thus, kallistatin appears to be a unique antioxidant and anti-inflammatory agent.

\section{Kallistatin Reduces Senescence in EPCs and Endothelial Cells}

EPCs are a subset of mononuclear cells derived from the bone marrow that have the ability to differentiate into mature endothelial cells [52]. Reduced EPC number is associated with defective proliferation and mobility as well as accelerated apoptosis and senescence [53]. Kallistatin depletion by neutralizing antibody injection augments glomerular endothelial cell loss and diminishes circulating EPC numbers [23, 24]. Excess oxidative stress or inflammation impairs the mobility and function of EPCs and increases cellular senescence [54, 55]. Indeed, recombinant human kallistatin treatment significantly inhibits TNF- $\alpha$-induced EPC senescence by blocking oxidative stress and reducing the senescent markers plasminogen activator inhibitor (PAI)-1, microRNA- (miR-) 21 , and p16 ${ }^{\mathrm{INK} 4 \mathrm{a}}$, while increasing telomerase activity [56]. The miR-34asirtuin 1 (SIRT1) axis is a key pathway in the aging process [57]. Kallistatin via its active site inhibits prosenescent miR34a expression and antagonizes miR-34a-mediated inhibition of the antioxidant enzymes SIRT1 and eNOS in EPCs [56]. As a deacetylase, SIRT1 stimulates antioxidant enzymes 


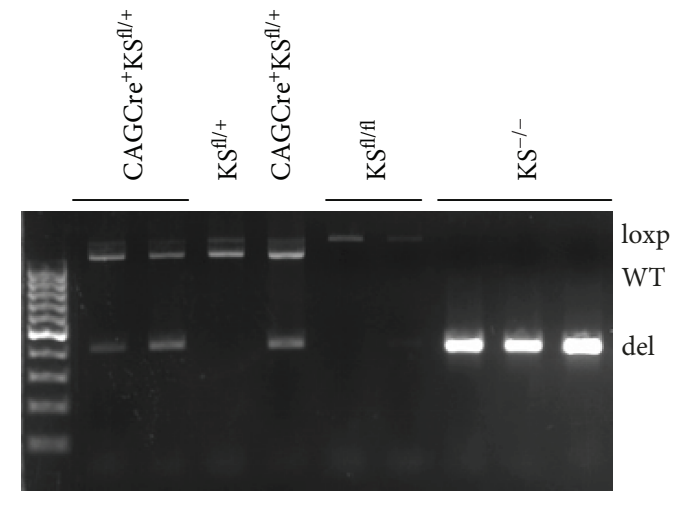

(a)

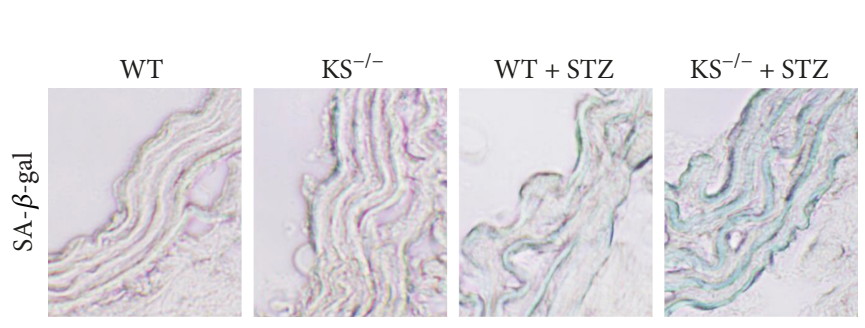

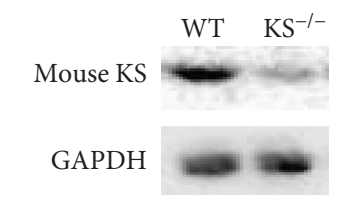

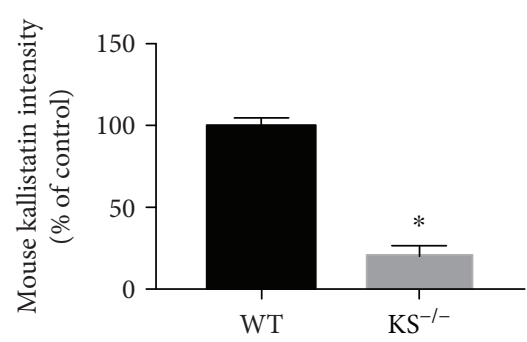

(b)

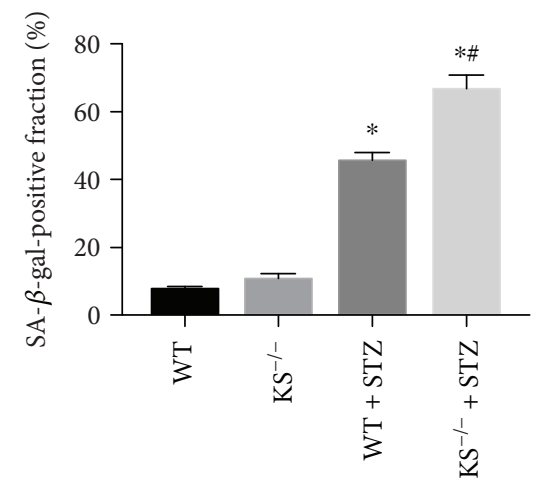

(c)

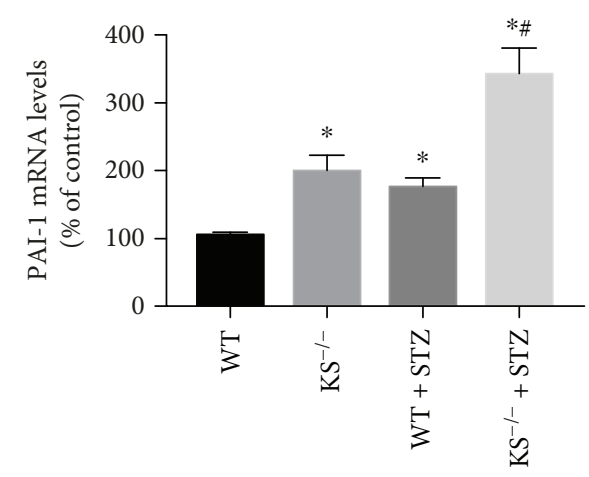

(d)

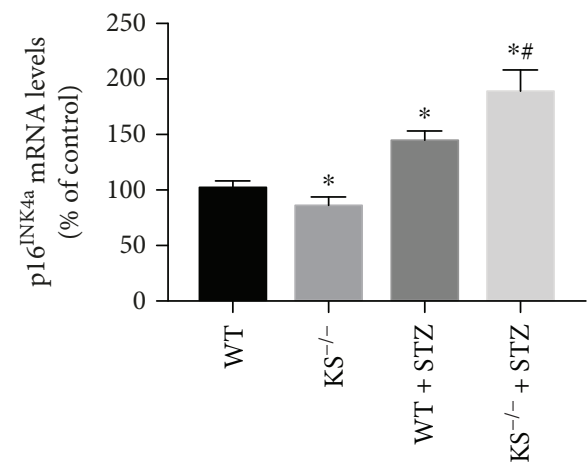

(e)

FIGURE 2: Systemic depletion of kallistatin exacerbates aortic senescence. (a) Kallistatin depletion identified by genotyping with the expression of loxp, wild-type (WT) and deletion (del) alleles. (b) Western blot analysis of mouse kallistatin expression in the kidney of WT mice and KS ${ }^{-1}$ - mice. (c) Representative images and quantitative analysis of $\beta$-gal staining of aorta sections from WT, $\mathrm{KS}^{-1-}$ mice with or without STZ treatment. ( $\mathrm{d}, \mathrm{e}) \mathrm{PAI}-1$ and P16 ${ }^{\mathrm{INK} 4 \mathrm{a}}$ mRNA levels in mouse aorta. Values are expressed as mean \pm SEM. $n=3$. ${ }^{*} P<0.05$ vs. the WT group, ${ }^{\#} P<0.05$ vs. the $\mathrm{WT}+\mathrm{STZ}$ group.

including eNOS, catalase, and superoxide dismutase (SOD), and eNOS through NO production stimulates SIRT1 enzymatic activity and inhibits NADPH oxidase activity $[58,59]$, leading to the attenuation of oxidative stress. Similarly, kallistatin exerts salutary effects in the setting of $\mathrm{H}_{2} \mathrm{O}_{2}$-induced endothelial senescence, oxidative stress, and inflammation, as indicated by reduced $\mathrm{p} 16^{\mathrm{INK} 4 \mathrm{a}}$, PAI-1, and miR-34a synthesis, NADPH oxidase activity/expression, vascular cell adhesion molecule- (VCAM-) 1 and intercellular adhesion molecule(ICAM-) 1 expression, and increased telomerase activity in endothelial cells [60]. Moreover, kallistatin via upregulating the endoprotective miRNA Let-7g coordinates Let-7gmodulated miR-34a-SIRT1-eNOS pathway and achieves antisenescent, antioxidant, and anti-inflammatory actions in endothelial cells [60]. Activation of SIRT1-eNOS signaling results in increased catalase and NO levels, thus suppressing oxidative vascular damage [60]. These combined studies reveal the mechanisms of kallistatin in protection against vascular injury by reducing cellular senescence in EPCs and endothelial cells. 

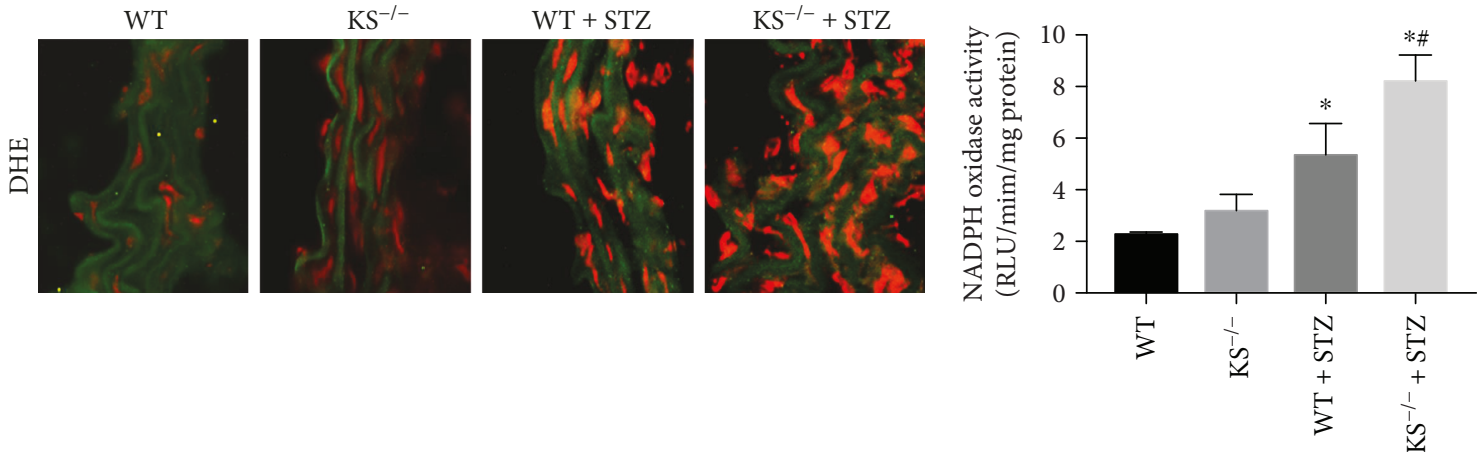

(a)

(b)

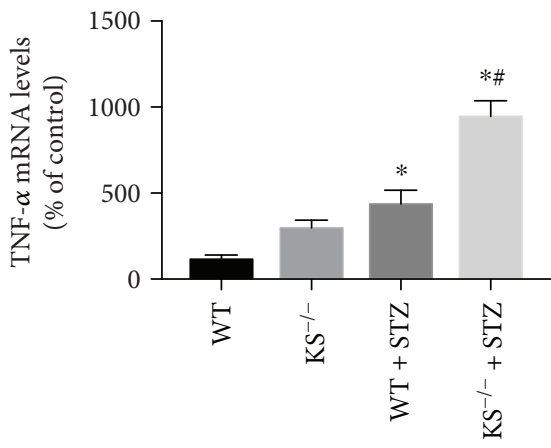

(c)
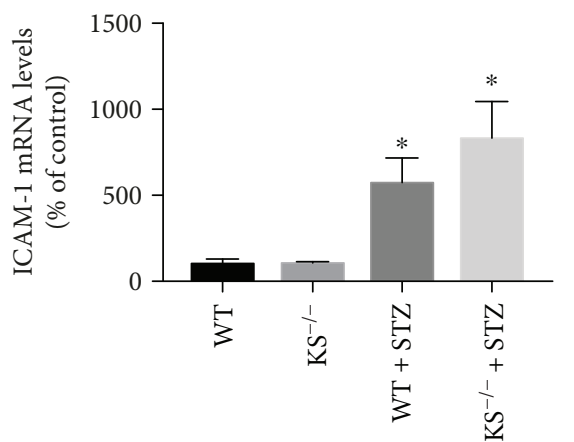

(d)

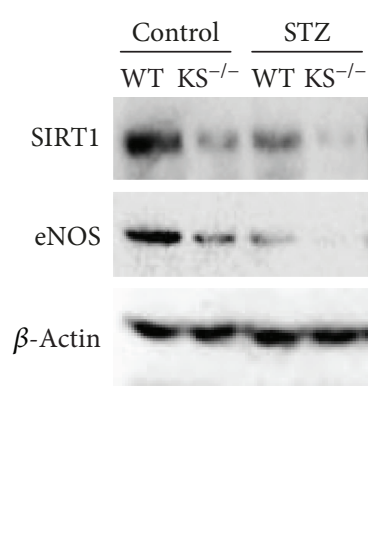

(e)

FIGURE 3: Systemic depletion of kallistatin exacerbates aortic oxidative stress and inflammation. (a) Representative images of superoxide formation in aortas from WT, $\mathrm{KS}^{-/-}$mice with or without STZ treatment, as indicated by red fluorescence dye DHE. Collagen fibers displayed green autofluorescence in the aorta. (b) NADPH oxidase in mouse aorta. (c) Representative western blot of SIRT1 and eNOS expression in mouse aorta. (d\&e) Inflammatory gene expression of TNF- $\alpha$ and ICAM-1 in mouse aorta. Values are expressed as mean \pm SEM. $n=3 .{ }^{*} P<0.05$ vs. WT group, ${ }^{\#} P<0.05$ vs. WT + STZ group.

\section{Kallistatin Treatment Attenuates Vascular Injury and Senescence in Animal Models}

As age advances, the alteration of vascular structure and function is intensified, such as endothelial senescence/rarefaction and increased oxidative stress and inflammation [61]. High blood glucose-induced vascular complications are manifested in diabetes [62]. The STZ-induced diabetic mouse is a popular model for studying vascular injury and aging $[63,64]$. Kallistatin protein treatment inhibits aortic senescence and superoxide formation in association with reduced miR-34a synthesis and increased SIRT1 and eNOS expression in STZ-induced diabetic mice [56]. Moreover, kallistatin administration significantly inhibits inflammatory gene expression, such as TNF- $\alpha$ and ICAM-1, in the aorta of STZ-induced diabetic mice (Figure 1), indicating the beneficial effect of kallistatin in diabetes-associated vascular senescence, oxidative stress, and inflammation. In addition to diabetes, kallistatin gene delivery suppresses aortic superoxide formation and glomerular capillary loss in salt-induced hypertensive rats [22]. Likewise, kallistatin treatment attenuates cardiac dysfunction, apoptosis, and inflammation in conjunction with decreased oxidative stress and increased eNOS and NO levels in animal models of hypertension and myocardial infarction and ischemia-reperfusion injury [18, 19, 22, 47, 65]. Furthermore, kallistatin gene delivery inhibits vascular leakage in mice provoked by $\mathrm{C} 5 \mathrm{a}$ and prevents arthritis-induced inflammation in rats [21]. Importantly, kallistatin is capable of improving oxidative stress-induced survival/aging at the organismal level in Caenorhabditis elegans by regulating the miR-34a-SIRT1 pathway [56]. Thus, as a potent antioxidant and anti-inflammatory agent, kallistatin could have a significant impact on vascular injury and senescence.

\section{Endothelial-Specific Depletion of Kallistatin Exacerbates Cellular Senescence, Oxidative Stress, and Inflammation in Mouse Endothelial Cells}

Endothelial-specific kallistatin knockout $\left(\mathrm{KS}^{\text {endo-/- }}\right)$ mice were generated by Cre-loxp recombination for exploring the role of endogenous kallistatin in diabetes-associated endothelial senescence, oxidative stress, and inflammation [60]. Mouse lung endothelial cells were isolated from 8week-old $\mathrm{KS}^{\text {endo-- }-}$ and wild-type (WT) mice with CD31 immunoselection and cultured as described [66]. Kallistatin deficiency in mouse lung endothelial cells displayed 


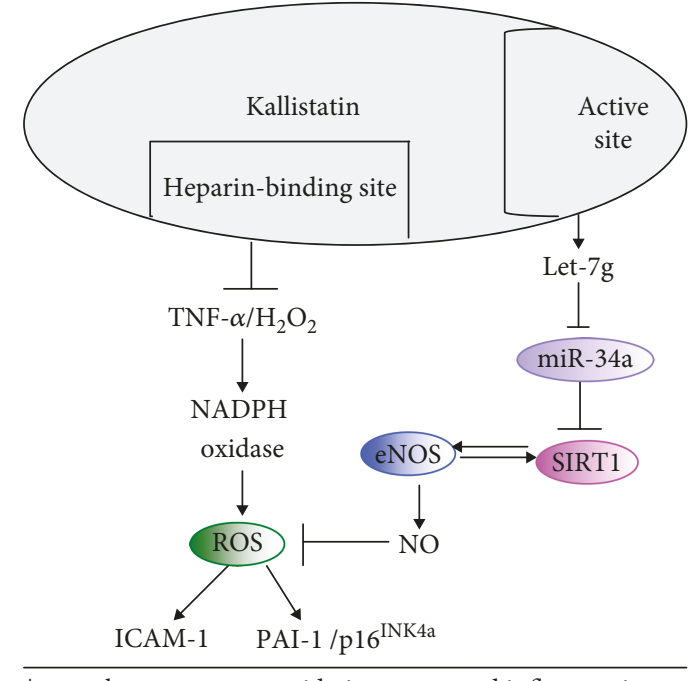

$\downarrow$ vascular senescence, oxidative stress, and inflammation

FIGURE 4: Signaling pathways of kallistatin in protection against vascular senescence, oxidative stress, and inflammation in EPCs and endothelial cells. Kallistatin via its heparin-binding site blocks TNF- $\alpha / \mathrm{H}_{2} \mathrm{O}_{2}$-modulated NADPH oxidase activity and ROS formation and thus ICAM-1 and PAI- $1 / \mathrm{p} 16^{\mathrm{INK} 4 \mathrm{a}}$ expression. Kallistatin through its active site stimulates Let-7g synthesis, to inhibit miR-34 and stimulate SIRT1-eNOS pathway, thereby elevating NO levels, further leading to reduced ROS levels.

aggravated senescence, oxidative stress, and inflammation, as evidenced by increases in senescence-associated (SA) $\beta$-gal activity, PAI- $1 / \mathrm{p} 16^{\mathrm{INK} 4 \mathrm{a}}$ synthesis, superoxide formation, and expression of the inflammatory genes ICAM-1, VCAM-1, and interleukin- (IL-) 6 [60]. Kallistatin deficiency in endothelial cells exacerbated oxidative stress-induced senescence, superoxide formation, NADPH oxidase activity, and inflammatory gene expression in endothelial cells, indicating a protective role of endogenous kallistatin in maintaining endothelial viability and function. Moreover, endothelial-specific depletion of kallistatin elevated senescence inducer miR-34a but reduced Let-7g and antioxidant genes, including SIRT1, eNOS, and catalase [60]. These findings support the notion that endogenous kallistatin acts as a protective molecule in endothelial senescence by inhibiting oxidative stress and inflammation.

\section{Systemic Depletion of Kallistatin Aggregates Vascular Injury, Senescence, Oxidative Stress, and Inflammation in Diabetic Mice}

Kallistatin depletion by neutralizing antibody injection aggravates aortic oxidative stress, capillary loss, and cardiovascular and renal remodeling, accompanied by elevated oxidative stress and inflammation in hypertensive rats $[23,24]$. To further investigate the role of endogenous kallistatin in vascular injury and senescence, we generated systemic deficiency of kallistatin $\left(\mathrm{KS}^{-1-}\right)$ mice by Cre-loxp recombination technology [60]. Briefly, female homozygous floxed $\mathrm{KS}^{\mathrm{fl} / \mathrm{fl}}$ mice were crossed with male CAGCre ${ }^{+}$ mice for two generations to obtain $\mathrm{CAGCre}{ }^{+} \mathrm{KS}^{\mathrm{fl} / \mathrm{fl}}$ mice.
Tamoxifen was injected in these mice to induce Cre recombinase activity. The genotyping result showed that only $\mathrm{KS}^{-/-}$ mice were positive for deletion allele expression and negative for loxp and WT allele expression (Figure 2(a)). Kallistatin depletion was further identified by reduced mouse kallistatin protein levels in western blot analysis in the kidney of $\mathrm{KS}^{-/-}$ mice, compared with WT mice (Figure 2(b)). In contrast with kallistatin's efficacy in aortic senescence and oxidative stress in STZ-induced diabetic mice [56], systemic depletion of kallistatin worsened vascular damage and senescence, characterized by aggravated aortic thickening, structural disarrangement, elevated SA- $\beta$-gal activity, and PAI- 1 and p16 ${ }^{\text {INK4a }}$ mRNA levels in the aorta of diabetic mice (Figures 2(c)-2(e)). Kallistatin depletion also worsened aortic collagen deposition, superoxide formation, and NADPH oxidase activity and elevated inflammatory gene expression, including TNF- $\alpha$ and ICAM-1 (Figures 3(a)-3(d)). Moreover, levels of the antioxidant proteins SIRT1 and eNOS were significantly reduced in the aorta of $\mathrm{KS}^{-1-}$ mice with STZinduced diabetes (Figure 3(e)), in contrast to the stimulatory effect of kallistatin administration on SIRT1 and eNOS expression [56]. These combined findings indicate that endogenous kallistatin is a protective agent against vascular injury and senescence.

\section{Conclusion}

These combined studies reveal a novel role of endogenous kallistatin in protection against vascular injury and senescence. Kallistatin reduces vascular injury and senescence by promoting the migration and function of EPCs. Exogenous kallistatin treatment attenuates aortic injury, senescence, oxidative stress, and inflammation in animal models, while kallistatin deficiency in endothelial-specific or general knockout mice exacerbates vascular injury, senescence, oxidative stress, and inflammation in primarily cultured mouse endothelial cells and diabetic mice. The signaling pathways by which kallistatin inhibits vascular senescence, oxidative stress, and inflammation in EPCs and endothelial cells are shown in Figure 4. Kallistatin is an endogenous protein with no cytotoxic effects. Therefore, kallistatin could potentially be used as an effective therapeutic regimen for aging-associated vascular disease in humans.

\section{Conflicts of Interest}

The authors declare that there are no conflicts of interest regarding the publication of this paper.

\section{Acknowledgments}

This study was supported by the National Institutes of Health grant HL118516.

\section{References}

[1] R. Dhingra and R. S. Vasan, “Age as a risk factor," The Medical Clinics of North America, vol. 96, no. 1, pp. 87-91, 2012.

[2] M. Kelly-Hayes, "Influence of age and health behaviors on stroke risk: lessons from longitudinal studies," Journal of the 
American Geriatrics Society, vol. 58, Supplement 2, pp. S325S328, 2010.

[3] M. C. White, D. M. Holman, J. E. Boehm, L. A. Peipins, M. Grossman, and S. Jane Henley, "Age and cancer risk: a potentially modifiable relationship," American Journal of Preventive Medicine, vol. 46, no. 3, pp. S7-15, 2014.

[4] J. Wu, S. Xia, B. Kalionis, W. Wan, and T. Sun, "The role of oxidative stress and inflammation in cardiovascular aging," BioMed Research International, vol. 2014, Article ID 615312, 13 pages, 2014.

[5] D. R. Seals, K. L. Jablonski, and A. J. Donato, "Aging and vascular endothelial function in humans," Clinical Science (London, England), vol. 120, no. 9, pp. 357-375, 2011.

[6] J. Case, D. A. Ingram, and L. S. Haneline, "Oxidative stress impairs endothelial progenitor cell function," Antioxidants \& Redox Signaling, vol. 10, no. 11, pp. 1895-1907, 2008.

[7] J. D. Erusalimsky, "Vascular endothelial senescence: from mechanisms to pathophysiology," Journal of Applied Physiology, vol. 106, no. 1, pp. 326-332, 2009.

[8] C. Besler, C. Doerries, G. Giannotti, T. F. Lüscher, and U. Landmesser, "Pharmacological approaches to improve endothelial repair mechanisms," Expert Review of Cardiovascular Therapy, vol. 6, no. 8, pp. 1071-1082, 2008.

[9] N. A. Mikirova, J. A. Jackson, R. Hunninghake et al., "Circulating endothelial progenitor cells: a new approach to anti-aging medicine?," Journal of Translational Medicine, vol. 7, no. 1, p. 106, 2009.

[10] C. Schmidt-Lucke, L. Rössig, S. Fichtlscherer et al., "Reduced number of circulating endothelial progenitor cells predicts future cardiovascular events: proof of concept for the clinical importance of endogenous vascular repair," Circulation, vol. 111, no. 22, pp. 2981-2987, 2005.

[11] C. Heiss, S. Keymel, U. Niesler, J. Ziemann, M. Kelm, and C. Kalka, "Impaired progenitor cell activity in age-related endothelial dysfunction," Journal of the American College of Cardiology, vol. 45, no. 9, pp. 1441-1448, 2005.

[12] K. X. Chai, D. C. Ward, J. Chao, and L. Chao, "Molecular cloning, sequence analysis, and chromosomal localization of the human protease inhibitor 4 (kallistatin) gene (PI4)," Genomics, vol. 23, no. 2, pp. 370-378, 1994.

[13] G. X. Zhou, L. Chao, and J. Chao, "Kallistatin: a novel human tissue kallikrein inhibitor. Purification, characterization, and reactive center sequence," The Journal of Biological Chemistry, vol. 267, no. 36, pp. 25873-25880, 1992.

[14] K. X. Chai, L. M. Chen, J. Chao, and L. Chao, "Kallistatin: a novel human serine proteinase inhibitor. Molecular cloning, tissue distribution, and expression in Escherichia coli," The Journal of Biological Chemistry, vol. 268, no. 32, pp. 2449824505, 1993.

[15] J. Chao, K. X. Chai, L. M. Chen et al., "Tissue kallikreinbinding protein is a serpin. I. Purification, characterization, and distribution in normotensive and spontaneously hypertensive rats," The Journal of Biological Chemistry, vol. 265, no. 27, pp. 16394-16401, 1990.

[16] J. Chao, D. M. Tillman, M. Y. Wang, H. S. Margolius, and L. Chao, "Identification of a new tissue-kallikrein-binding protein," The Biochemical Journal, vol.239, no. 2, pp.325-331, 1986.

[17] P. Li, G. Bledsoe, Z. R. Yang, H. Fan, L. Chao, and J. Chao, "Human kallistatin administration reduces organ injury and improves survival in a mouse model of polymicrobial sepsis," Immunology, vol. 142, no. 2, pp. 216-226, 2014.
[18] B. Shen, M. Hagiwara, Y. Y. Yao, L. Chao, and J. Chao, "Salutary effect of kallistatin in salt-induced renal injury, inflammation, and fibrosis via antioxidative stress," Hypertension, vol. 51, no. 5, pp. 1358-1365, 2008.

[19] J. Chao, H. Yin, Y. Y. Yao, B. Shen, R. S. Smith Jr, and L. Chao, "Novel role of kallistatin in protection against myocardial ischemia-reperfusion injury by preventing apoptosis and inflammation," Human Gene Therapy, vol. 17, no. 12, pp. 1201-1213, 2006.

[20] J. Chao, J. N. Stallone, Y. M. Liang, L. M. Chen, D. Z. Wang, and L. Chao, "Kallistatin is a potent new vasodilator," The Journal of Clinical Investigation, vol. 100, no. 1, pp. 11-17, 1997.

[21] H. Yin, L. Gao, B. Shen, L. Chao, and J. Chao, "Kallistatin inhibits vascular inflammation by antagonizing tumor necrosis factor- $\alpha$-induced nuclear factor $\kappa \mathrm{B}$ activation," Hypertension, vol. 56, no. 2, pp. 260-267, 2010.

[22] B. Shen, L. Gao, Y. T. Hsu et al., "Kallistatin attenuates endothelial apoptosis through inhibition of oxidative stress and activation of Akt-eNOS signaling," American Journal of Physiology. Heart and Circulatory Physiology, vol. 299, no. 5, pp. H1419-H1427, 2010.

[23] L. Gao, P. Li, J. Zhang et al., "Novel role of kallistatin in vascular repair by promoting mobility, viability, and function of endothelial progenitor cells," Journal of the American Heart Association, vol. 3, no. 5, article e001194, 2014.

[24] Y. Liu, G. Bledsoe, M. Hagiwara, B. Shen, L. Chao, and J. Chao, "Depletion of endogenous kallistatin exacerbates renal and cardiovascular oxidative stress, inflammation, and organ remodeling," American Journal of Physiology. Renal Physiology, vol. 303, no. 8, pp. F1230-F1238, 2012.

[25] H. Zhu, J. Chao, A. Raed et al., "Plasma kallistatin is associated with leukocyte telomere length in young African Americans," The FASEB Journal, vol. 30, 1_Supplement, p. 625.1, 2016.

[26] T. Minamino, H. Miyauchi, T. Yoshida, K. Tateno, T. Kunieda, and I. Komuro, "Vascular cell senescence and vascular aging," Journal of Molecular and Cellular Cardiology, vol. 36, no. 2, pp. 175-183, 2004.

[27] M. Vasa, K. Breitschopf, A. M. Zeiher, and S. Dimmeler, "Nitric oxide activates telomerase and delays endothelial cell senescence," Circulation Research, vol. 87, no. 7, pp. 540$542,2000$.

[28] J. Chao and L. Chao, "Biochemistry, regulation and potential function of kallistatin," Biological Chemistry Hoppe-Seyler, vol. 376, no. 12, pp. 705-713, 1995.

[29] J. Chao, A. Schmaier, L. M. Chen, Z. Yang, and L. Chao, "Kallistatin, a novel human tissue kallikrein inhibitor: levels in body fluids, blood cells, and tissues in health and disease," The Journal of Laboratory and Clinical Medicine, vol. 127, no. 6, pp. 612-620, 1996.

[30] W. C. Wolf, R. A. Harley, D. Sluce, L. Chao, and J. Chao, "Localization and expression of tissue kallikrein and kallistatin in human blood vessels," Journal of Histochemistry \& Cytochemistry, vol. 47, no. 2, pp. 221-228, 1999.

[31] J. Chao, G. Bledsoe, and L. Chao, "Protective role of kallistatin in vascular and organ injury," Hypertension, vol. 68, no. 3, pp. 533-541, 2016.

[32] M. A. De Groote, D. G. Sterling, T. Hraha et al., "Discovery and validation of a six-marker serum protein signature for the diagnosis of active pulmonary tuberculosis," Journal of Clinical Microbiology, vol. 55, no. 10, pp. 30573071, 2017. 
[33] W. C. Lin, S. L. Lu, C. F. Lin et al., "Plasma kallistatin levels in patients with severe community-acquired pneumonia," Critical Care, vol. 17, no. 1, p. R27, 2013.

[34] J. Chao, P. Li, and L. Chao, "Kallistatin suppresses cancer development by multi-factorial actions," Critical Reviews in Oncology/Hematology, vol. 113, pp. 71-78, 2017.

[35] W. C. Lin, C. W. Chen, L. Chao, J. Chao, and Y. S. Lin, "Plasma kallistatin in critically ill patients with severe sepsis and septic shock," PLoS One, vol. 12, no. 5, article e0178387, 2017.

[36] H. Zhu, J. Chao, I. Kotak et al., "Plasma kallistatin is associated with adiposity and cardiometabolic risk in apparently healthy African American adolescents," Metabolism, vol. 62, no. 5, pp. 642-646, 2013.

[37] J. X. Ma, L. P. King, Z. Yang, R. K. Crouch, L. Chao, and J. Chao, "Kallistatin in human ocular tissues: reduced levels in vitreous fluids from patients with diabetic retinopathy," Current Eye Research, vol. 15, no. 11, pp. 1117-1123, 1996.

[38] H. C. Hatcher, J. X. Ma, J. Chao, L. Chao, and A. Ottlecz, "Kallikrein-binding protein levels are reduced in the retinas of streptozotocin-induced diabetic rats," Investigative Ophthalmology \& Visual Science, vol. 38, no. 3, pp. 658-664, 1997.

[39] A. J. Jenkins, J. D. McBride, A. S. Januszewski et al., "Increased serum kallistatin levels in type 1 diabetes patients with vascular complications," Journal of Angiogenesis Research, vol. 2, no. 1, p. $19,2010$.

[40] L. M. Chen, L. Chao, and J. Chao, "Beneficial effects of kallikrein-binding protein in transgenic mice during endotoxic shock," Life Sciences, vol. 60, no. 17, pp. 1431-1435, 1997.

[41] X. Zhi, L. Lin, S. Yang et al., " $\beta$ II-Spectrin (SPTBN1) suppresses progression of hepatocellular carcinoma and Wnt signaling by regulation of Wnt inhibitor kallistatin," Hepatology, vol. 61, no. 2, pp. 598-612, 2015.

[42] J. Chao and L. Chao, "A major difference of kallikreinbinding protein in spontaneously hypertensive versus normotensive rats," Journal of Hypertension, vol. 6, no. 7, pp. 551-558, 1988.

[43] J. Chao, Y. Guo, P. Li, and L. Chao, “Opposing effects of oxygen regulation on kallistatin expression: kallistatin as a novel mediator of oxygen-induced HIF-1-eNOS-NO pathway," Oxidative Medicine and Cellular Longevity, vol. 2017, Article ID 5262958, 8 pages, 2017.

[44] A. Stadnicki, U. Mazurek, M. Gonciarz et al., "Immunolocalization and expression of kallistatin and tissue kallikrein in human inflammatory bowel disease," Digestive Diseases and Sciences, vol. 48, no. 3, pp. 615-623, 2003.

[45] C. Urso and G. Caimi, "Oxidative stress and endothelial dysfunction,” Minerva Medica, vol. 102, no. 1, pp. 59-77, 2011.

[46] J. A. Woods, K. R. Wilund, S. A. Martin, and B. M. Kistler, "Exercise, inflammation and aging," Aging and Disease, vol. 3, no. 1, pp. 130-140, 2012.

[47] L. Gao, H. Yin, R. S Smith, L. Chao, and J. Chao, "Role of kallistatin in prevention of cardiac remodeling after chronic myocardial infarction," Laboratory Investigation, vol. 88, no. 11, pp. 1157-1166, 2008.

[48] P. Li, Y. Guo, G. Bledsoe et al., "Kallistatin treatment attenuates lethality and organ injury in mouse models of established sepsis," Critical Care, vol. 19, no. 1, p. 200, 2015.

[49] W. H. Yiu, D. W. L. Wong, H. J. Wu et al., "Kallistatin protects against diabetic nephropathy in $\mathrm{db} / \mathrm{db}$ mice by suppressing
AGE-RAGE-induced oxidative stress," Kidney International, vol. 89, no. 2, pp. 386-398, 2016.

[50] H. Fujii, K. Ichimori, K. Hoshiai, and H. Nakazawa, "Nitric oxide inactivates NADPH oxidase in pig neutrophils by inhibiting its assembling process," The Journal of Biological Chemistry, vol. 272, no. 52, pp. 32773-32778, 1997.

[51] B. Shen, R. S. Smith Jr., Y. T. Hsu, L. Chao, and J. Chao, "Kruppel-like factor 4 is a novel mediator of kallistatin in inhibiting endothelial inflammation via increased endothelial nitricoxide synthase expression," The Journal of Biological Chemistry, vol. 284, no. 51, pp. 35471-35478, 2009.

[52] M. C. Yoder, "Human endothelial progenitor cells," Cold Spring Harbor Perspectives in Medicine, vol. 2, no. 7, article a006692, 2012.

[53] T. Imanishi, H. Tsujioka, and T. Akasaka, "Endothelial progenitor cells dysfunction and senescence: contribution to oxidative stress," Current Cardiology Reviews, vol. 4, no. 4, pp. 275-286, 2008.

[54] T. Imanishi, T. Hano, and I. Nishio, “Angiotensin II accelerates endothelial progenitor cell senescence through induction of oxidative stress," Journal of Hypertension, vol. 23, no. 1, pp. 97-104, 2005.

[55] K. Sugamura and J. F. Keaney Jr, "Reactive oxygen species in cardiovascular disease," Free Radical Biology and Medicine, vol. 51, no. 5, pp. 978-992, 2011.

[56] Y. Guo, P. Li, L. Gao et al., "Kallistatin reduces vascular senescence and aging by regulating microRNA-34a-SIRT1 pathway," Aging Cell, vol. 16, no. 4, pp. 837-846, 2017.

[57] T. Ito, S. Yagi, and M. Yamakuchi, "MicroRNA-34a regulation of endothelial senescence," Biochemical and Biophysical Research Communications, vol. 398, no. 4, pp. 735-740, 2010.

[58] M. Potente and S. Dimmeler, "NO targets SIRT1: a novel signaling network in endothelial senescence," Arteriosclerosis, Thrombosis, and Vascular Biology, vol. 28, no. 9, pp. 15771579, 2008.

[59] S. Selemidis, G. Dusting, H. Peshavariya, B. Kempharper, and G. Drummond, "Nitric oxide suppresses NADPH oxidasedependent superoxide production by S-nitrosylation in human endothelial cells," Cardiovascular Research, vol. 75, no. 2, pp. 349-358, 2007.

[60] Y. Guo, L. Chao, and J. Chao, "Kallistatin attenuates endothelial senescence by modulating Let-7g-mediated miR-34aSIRT1-eNOS pathway," Journal of Cellular and Molecular Medicine, vol. 22, no. 9, pp. 4387-4398, 2018.

[61] A. J. Donato, R. G. Morgan, A. E. Walker, and L. A. Lesniewski, "Cellular and molecular biology of aging endothelial cells," Journal of Molecular and Cellular Cardiology, vol. 89, Part B, pp. 122-135, 2015.

[62] C. Rask-Madsen and G. L. King, "Vascular complications of diabetes: mechanisms of injury and protective factors," Cell Metabolism, vol. 17, no. 1, pp. 20-33, 2013.

[63] G. Prévost, H. Bulckaen, C. Gaxatte et al., "Structural modifications in the arterial wall during physiological aging and as a result of diabetes mellitus in a mouse model: are the changes comparable?," Diabetes \& Metabolism, vol. 37, no. 2, pp. 106-111, 2011.

[64] H. Ota, M. Eto, M. R. Kano et al., "Induction of endothelial nitric oxide synthase, SIRT1, and catalase by statins inhibits endothelial senescence through the Akt pathway," Arteriosclerosis, Thrombosis, and Vascular Biology, vol. 30, no. 11, pp. 2205-2211, 2010. 
[65] J. Chao and L. Chao, "Kallistatin in blood pressure regulation transgenic and somatic gene delivery studies," Trends in Cardiovascular Medicine, vol. 7, no. 8, pp. 307-311, 1997.

[66] R. S. Alphonse, A. Vadivel, S. Zhong et al., "The isolation and culture of endothelial colony-forming cells from human and rat lungs," Nature Protocols, vol. 10, no. 11, pp. 1697-1708, 2015. 


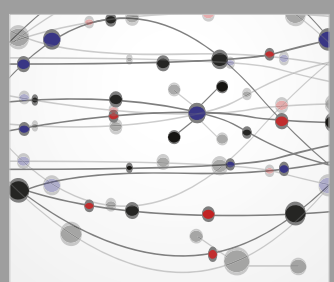

The Scientific World Journal
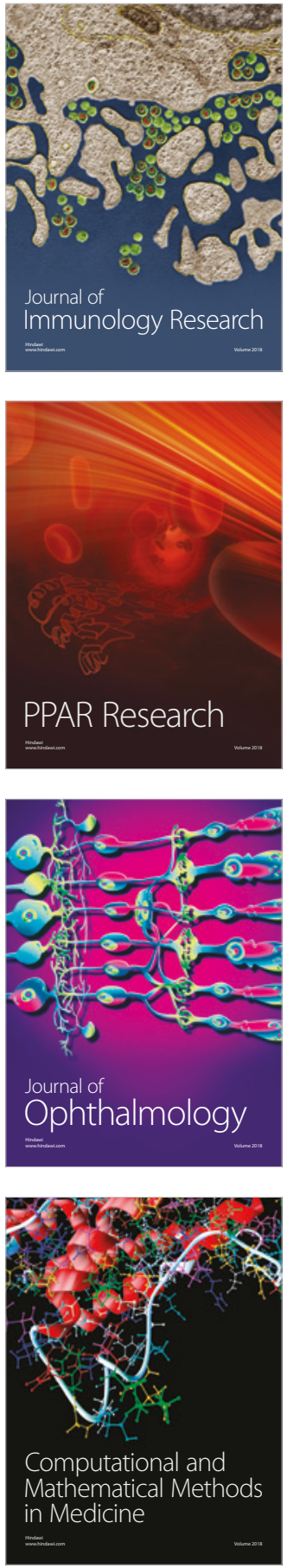

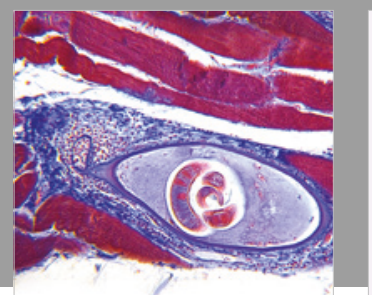

Gastroenterology Research and Practice

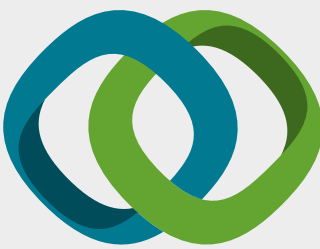

\section{Hindawi}

Submit your manuscripts at

www.hindawi.com
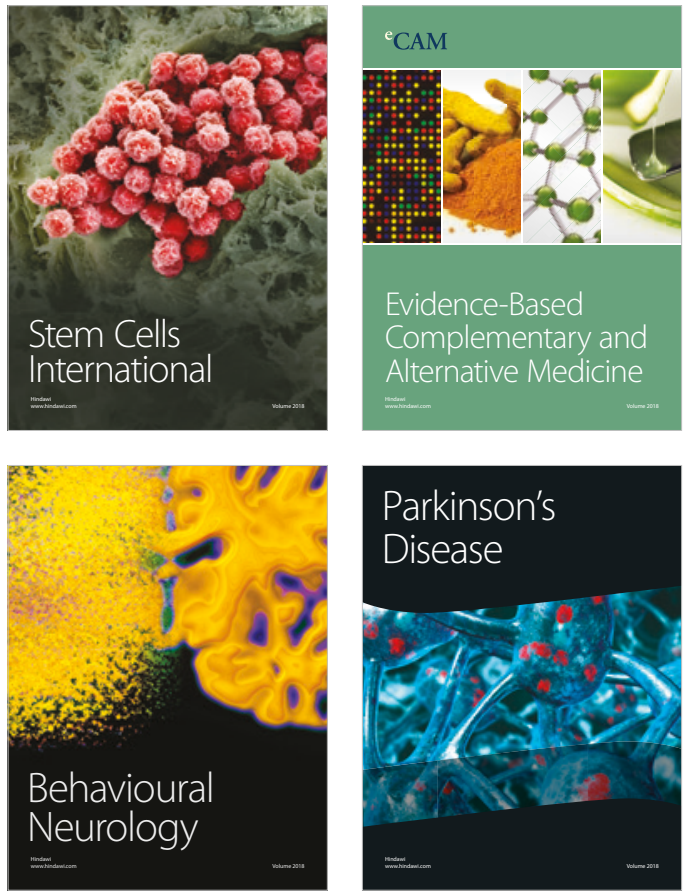

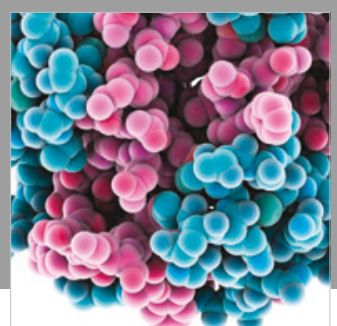

ournal of

Diabetes Research

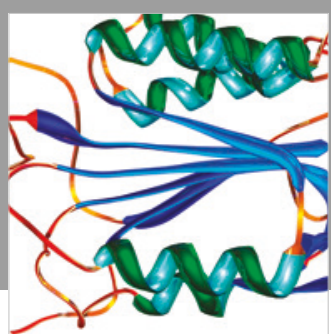

Disease Markers
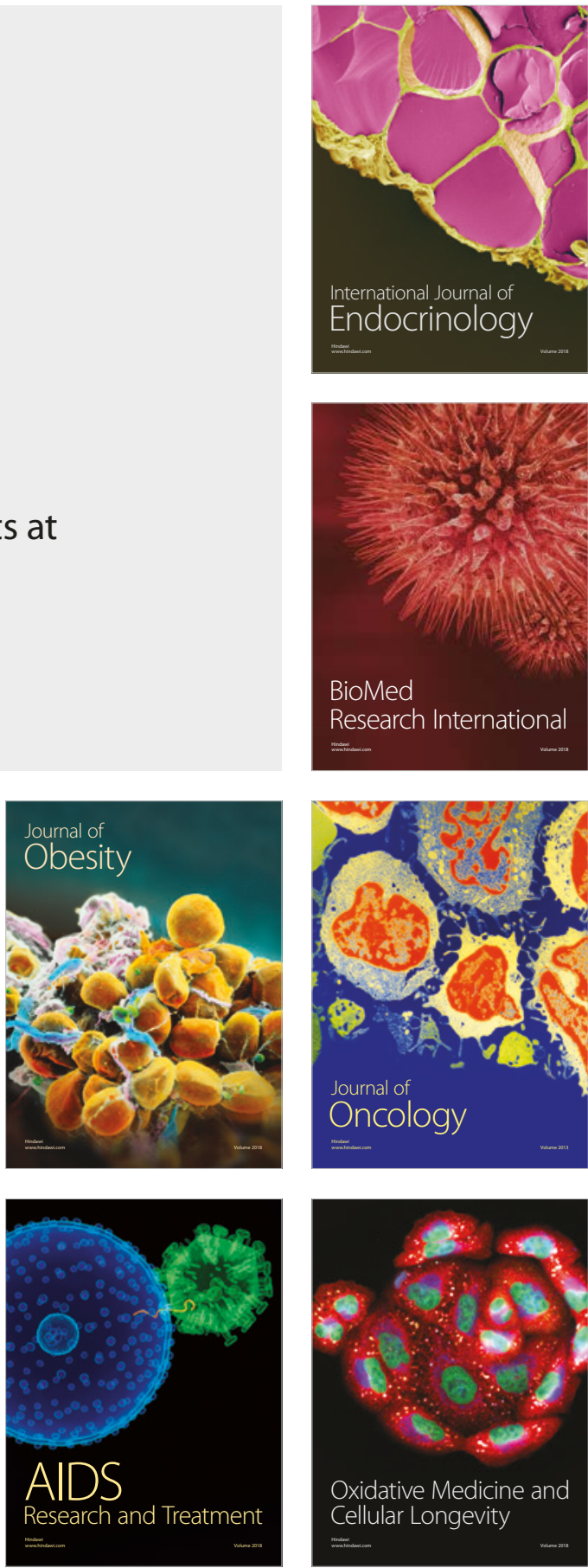